\title{
Unrestricted Cesàro summability of $d$-dimensional Fourier series and Lebesgue points
}

\author{
FERENC WEISZ*
}

ABSTRACT. We generalize the classical Lebesgue's theorem to multi-dimensional functions. We prove that the Cesàro means of the Fourier series of the multi-dimensional function $f \in L_{1}(\log L)^{d-1}\left(\mathbb{T}^{d}\right) \supset L_{p}\left(\mathbb{T}^{d}\right)(1<p \leq \infty)$ converge to $f$ at each strong Lebesgue point.

Keywords: Cesàro summability, strong Hardy-Littlewood maximal function, strong Lebesgue points.

2020 Mathematics Subject Classification: 42B08, 42A38, 42A24, 42B25.

Dedicated to Professor Francesco Altomare, on occasion of his 70th birthday, with esteem and friendship.

\section{INTRODUCTION}

In 1904, Fejér [3] investigated the arithmetic means of the partial sums of the trigonometric Fourier series of a one-dimensional function $f$, the so called Fejér means and proved that if the left and right limits $f(x-0)$ and $f(x+0)$ exist at a point $x$, then the Fejér means

$$
\sigma_{n} f(x):=\sum_{k=-n}^{n}\left(1-\frac{|k|}{n}\right) \widehat{f}(k) e^{\imath k x}
$$

converge to $(f(x-0)+f(x+0)) / 2$. Here, $\widehat{f}(k)$ denotes the $k$-th Fourier coefficient. One year later, Lebesgue [11] extended this theorem and obtained that every one-dimensional integrable function is Fejér summable at each Lebesgue point, thus almost everywhere. Some years later, M. Riesz [15] generalized this theorem for the Cesàro means of one-dimensional integrable functions (the definition can be found later).

The Cesàro summability is investigated in a great number of papers (see e.g. Gát $[4,5,6]$, Goginava [7, 8, 9], Simon [17, 18], Nagy, Persson, Tephnadze and Wall [13, 14] and Weisz $[19,20])$. In this short note, we generalize the result of Lebesgue and Riesz to this summability of multi-dimensional functions. We generalize the Lebesgue points and introduce the so called strong Lebesgue points. It is known that almost every point is a strong Lebesgue point of $f \in L_{1}(\log L)^{d-1}\left(\mathbb{T}^{d}\right)$. We introduce the strong Hardy-Littlewood maximal function $M_{s} f$ and show that the Cesàro means of $f \in L_{1}(\log L)^{d-1}\left(\mathbb{T}^{d}\right)$ can be estimated by $M_{s} f$ pointwise. Our main result is the following. If $M_{s} f(x)$ is finite and $x$ is a strong Lebesgue point of $f \in L_{1}(\log L)^{d-1}\left(\mathbb{T}^{d}\right)$, then

$$
\lim _{n \rightarrow \infty} \sigma_{n}^{\alpha} f(x)=f(x)
$$


where $\sigma_{n}^{\alpha} f$ denotes the $n$-th Cesàro means of the Fourier series of $f$. This implies the convergence of the Cesàro means almost everywhere as well as covers the one-dimensional results mentioned above. Note that $L_{1}(\log L)^{d-1}\left(\mathbb{T}^{d}\right) \supset L_{p}\left(\mathbb{T}^{d}\right)$ with $1<p \leq \infty$. The results are not true for $L_{1}\left(\mathbb{T}^{d}\right)$ if $d>1$. Similar theorems are known for the $\theta$-means generated by a single function $\theta$ (see Feichtinger and Weisz [2] and the references therein). However, those results and proofs do not contain the results for Cesàro means. For the multi-dimensional Cesàro means, we need new ideas.

\section{STRONG MAXIMAL FUNCTION AND STRONG LEBESGUE POINTS}

Let us fix $d \in \mathbb{N}$. For a set $\mathbb{Y} \neq \emptyset$, let $\mathbb{Y}^{d}$ be its Cartesian product $\mathbb{Y} \times \ldots \times \mathbb{Y}$ taken with itself $d$ times. We briefly write $L_{p}\left(\mathbb{T}^{d}\right)$ instead of the $L_{p}\left(\mathbb{T}^{d}, \lambda\right)$ space equipped with the norm

$$
\|f\|_{p}:=\left(\int_{\mathbb{T}^{d}}|f|^{p} d \lambda\right)^{1 / p} \quad(1 \leq p<\infty),
$$

with the usual modification for $p=\infty$, where $\lambda$ is the Lebesgue measure. We identify the torus $\mathbb{T}$ with $[-\pi, \pi]$. Set $\log ^{+} u:=\max (0, \log u)$. For $k \in \mathbb{N}$ and $1 \leq p<\infty$, a measurable function $f$ is in the set $L_{p}(\log L)^{k}\left(\mathbb{T}^{d}\right)$ if

$$
\|f\|_{L_{p}(\log L)^{k}}:=\left(\int_{\mathbb{T}^{d}}|f|^{p}\left(\log ^{+}|f|\right)^{k} d \lambda\right)^{1 / p}<\infty .
$$

For $k=0$, we get back the $L_{p}\left(\mathbb{T}^{d}\right)$ spaces. We have for all $k \in \mathbb{P}$ and $1 \leq p<r \leq \infty$ that

$$
L_{p}\left(\mathbb{T}^{d}\right) \supset L_{p}(\log L)^{k-1}\left(\mathbb{T}^{d}\right) \supset L_{p}(\log L)^{k}\left(\mathbb{T}^{d}\right) \supset L_{r}\left(\mathbb{T}^{d}\right) .
$$

For $f \in L_{1}\left(\mathbb{T}^{d}\right)$, the strong Hardy-Littlewood maximal function is defined by

$$
M_{s} f(x):=\sup _{h \in \mathbb{R}_{+}^{d}} \frac{1}{\prod_{j=1}^{d}\left(2 h_{j}\right)} \int_{-h_{1}}^{h_{1}} \cdots \int_{-h_{d}}^{h_{d}}|f(x-t)| d t .
$$

For $d>1$, it is known that there is a function $f \in L_{1}\left(\mathbb{T}^{d}\right)$ such that $M_{s} f=\infty$ almost everywhere (see Jessen, Marcinkiewicz and Zygmund [10] and Saks [16]). Thus, in contrary to the onedimensional case, $M_{s}$ cannot be of weak type $(1,1)$ if $d>1$. However, we know the following weak type inequality. If $f \in L(\log L)^{d-1}\left(\mathbb{T}^{d}\right)$, then

$$
\sup _{\rho>0} \rho \lambda\left(M_{s} f>\rho\right) \leq C+C\left\||f|\left(\log ^{+}|f|\right)^{d-1}\right\|_{1} \text {. }
$$

Moreover, for $1<p \leq \infty$, we have

$$
\left\|M_{s} f\right\|_{p} \leq C_{p}\|f\|_{p} \quad\left(f \in L_{p}\left(\mathbb{T}^{d}\right)\right) .
$$

In this paper, the constants $C$ and $C_{p}$ may vary from line to line. If $f \in L_{1}(\log L)^{d-1}\left(\mathbb{T}^{d}\right)$, then

$$
\lim _{h \rightarrow 0} \frac{1}{\prod_{j=1}^{d}\left(2 h_{j}\right)} \int_{-h_{1}}^{h_{1}} \cdots \int_{-h_{d}}^{h_{d}} f(x-t) d t=f(x)
$$

for almost every $x \in \mathbb{T}^{d}$. Here $h \rightarrow 0$ means that $h_{j} \rightarrow 0$ for all $j=1, \ldots, d$. Note that this result does not hold for all $f \in L_{1}\left(\mathbb{T}^{d}\right)$ if $d>1$ (see Jessen, Marcinkiewicz and Zygmund [10] and Saks [16]).

Motivated by this convergence result, a point $x \in \mathbb{T}^{d}$ is called a strong Lebesgue point of $f \in L_{p}\left(\mathbb{T}^{d}\right)$ if

$$
\lim _{h \rightarrow 0} \frac{1}{\prod_{j=1}^{d}\left(2 h_{j}\right)} \int_{-h_{1}}^{h_{1}} \cdots \int_{-h_{d}}^{h_{d}}|f(x-t)-f(x)| d t=0 .
$$


Theorem 2.1. Almost every point $x \in \mathbb{T}^{d}$ is a strong Lebesgue point of $f \in L_{1}(\log L)^{d-1}\left(\mathbb{T}^{d}\right)$.

This is not true for $f \in L_{1}\left(\mathbb{T}^{d}\right)$ if $d>1$. Note that $L_{1}(\log L)^{d-1}\left(\mathbb{T}^{d}\right) \supset L_{p}\left(\mathbb{T}^{d}\right)$ for all $1<p \leq$ $\infty$. For the results of this section, see Chang and Fefferman [1], Zygmund [21] or Weisz [19, 20].

\section{Rectangular CesÀro SUMmability}

For $\alpha \neq-1,-2, \ldots$ and $n \in \mathbb{N}$, let

$$
A_{n}^{\alpha}:=\left(\begin{array}{c}
n+\alpha \\
n
\end{array}\right)=\frac{(\alpha+1)(\alpha+2) \cdots(\alpha+n)}{n !} .
$$

Then $A_{0}^{\alpha}=1, A_{n}^{0}=1$ and $A_{n}^{1}=n+1(n \in \mathbb{N})$. The $k$-th Fourier coefficient of a $d$-dimensional integrable function $f \in L_{1}\left(\mathbb{T}^{d}\right)$ is defined by

$$
\widehat{f}(k)=\frac{1}{(2 \pi)^{d}} \int_{\mathbb{T}^{d}} f(x) e^{-\imath k \cdot x} d x \quad\left(k \in \mathbb{Z}^{d}\right),
$$

where $u \cdot x:=\sum_{k=1}^{d} u_{k} x_{k}$ for $x=\left(x_{1}, \ldots, x_{d}\right) \in \mathbb{R}^{d}$ and $u=\left(u_{1}, \ldots, u_{d}\right) \in \mathbb{R}^{d}$. Since the Fourier series of $f$ has bad convergence properties (see e.g. Weisz [20]), we consider the Cesàro summability.

Let $f \in L_{1}\left(\mathbb{T}^{d}\right), n=\left(n_{1}, \ldots, n_{d}\right) \in \mathbb{N}^{d}$ and $\alpha=\left(\alpha_{1}, \ldots, \alpha_{d}\right) \in \mathbb{R}_{+}^{d}$. The $n$-th rectangular Cesàro means $\sigma_{n}^{\alpha} f$ of the Fourier series of $f$ and the Cesàro kernel $K_{n}^{\alpha}$ are introduced by

$$
\sigma_{n}^{\alpha} f(x):=\frac{1}{\prod_{i=1}^{d} A_{n_{i}-1}^{\alpha}} \sum_{\left|k_{1}\right| \leq n_{1}} \cdots \sum_{\left|k_{d}\right| \leq n_{d}} \prod_{i=1}^{d} A_{n_{i}-1-\left|k_{i}\right|}^{\alpha} \widehat{f}(k) e^{\imath k \cdot x}
$$

and

$$
K_{n}^{\alpha}(t):=\frac{1}{\prod_{i=1}^{d} A_{n_{i}-1}^{\alpha}} \sum_{\left|k_{1}\right| \leq n_{1}} \cdots \sum_{\left|k_{d}\right| \leq n_{d}} \prod_{i=1}^{d} A_{n_{i}-1-\left|k_{i}\right|}^{\alpha} e^{\imath k \cdot t},
$$

respectively. It is easy to see that

$$
\sigma_{n}^{\alpha} f(x)=\frac{1}{(2 \pi)^{d}} \int_{\mathbb{T}^{d}} f(x-t) K_{n}^{\alpha}(t) d t
$$

and

$$
K_{n}^{\alpha}=K_{n_{1}}^{\alpha_{1}} \otimes \cdots \otimes K_{n_{d}}^{\alpha_{d}},
$$

where the functions $K_{n_{i}}^{\alpha_{i}}$ are the one-dimensional Cesàro kernels. The Cesàro means are also called $(C, \alpha)$-means. If all $\alpha_{i}=1$, then we get back the rectangular Fejér means. For the onedimensional Cesàro kernels, it is known (see Zygmund [21]) that

$$
K_{n}^{\alpha}(t) \leq C \min \left(n, \frac{1}{n^{\alpha}|t|^{\alpha+1}}\right)
$$

and

$$
\sup _{n \in \mathbb{N}} \int_{\mathbb{T}}\left|K_{n}^{\alpha}\right| d \lambda \leq C,
$$

where $n \in \mathbb{N}, 0<\alpha \leq 1$ and $t \in(-\pi, \pi)$. 


\section{UNRESTRICTED CONVERGENCE AT LEBESGUE POINTS}

Before proving the main results of this paper, we introduce the Herz space $E_{\infty}\left(\mathbb{R}^{d}\right)$ with the norm

$$
\|f\|_{E_{\infty}}:=\sum_{k_{1}=-\infty}^{\infty} \cdots \sum_{k_{d}=-\infty}^{\infty} 2^{k_{1}+\ldots+k_{d}}\left\|f 1_{P_{k}}\right\|_{\infty}<\infty
$$

where

$$
P_{k}:=P_{k_{1}} \times \cdots \times P_{k_{d}} \quad\left(k \in \mathbb{Z}^{d}\right)
$$

and

$$
P_{i}=\left\{x \in \mathbb{R}: 2^{i-1} \pi \leq|x|<2^{i} \pi\right\} \quad(i \in \mathbb{Z}) .
$$

Obviously, $L_{1}\left(\mathbb{R}^{d}\right) \supset E_{\infty}\left(\mathbb{R}^{d}\right)$. First, we will estimate pointwise the maximal operator

$$
\sigma_{*}^{\alpha} f:=\sup _{n \in \mathbb{N}^{d}}\left|\sigma_{n}^{\alpha} f\right|
$$

by the strong Hardy-Littlewood maximal function. To this end, we introduce the functions

$$
h^{\alpha_{j}}(t):=\min \left\{1,|t|^{-\alpha_{j}-1}\right\} \quad(t \in \mathbb{R})
$$

and

$$
h^{\alpha}:=h^{\alpha_{1}} \otimes \cdots \otimes h^{\alpha_{d}} .
$$

We get from (3.3) that

$$
\frac{1}{n_{j}}\left|\left(1_{(-\pi, \pi)} K_{n_{j}}^{\alpha_{j}}\right)\left(\frac{t}{n_{j}}\right)\right| \leq \frac{C}{n_{j}} \min \left\{n_{j}, \frac{n_{j}}{|t|^{\alpha_{j}+1}}\right\}=C h^{\alpha_{j}}(t) \quad(t \in \mathbb{R}) .
$$

It is easy to see that

$$
\left\|h^{\alpha}\right\|_{E_{\infty}\left(\mathbb{R}^{d}\right)}=\prod_{j=1}^{d}\left\|h^{\alpha_{j}}\right\|_{E_{\infty}(\mathbb{R})} \leq C_{\alpha} .
$$

Theorem 4.2. Suppose that $0<\alpha_{j} \leq 1$ for all $j=1, \ldots, d$. If $f \in L_{1}\left(\mathbb{T}^{d}\right)$ and $x \in \mathbb{T}^{d}$, then

$$
\sigma_{*}^{\alpha} f(x) \leq C M_{s} f(x) .
$$

Proof. Observe that

$$
\begin{aligned}
\left|\sigma_{n}^{\alpha} f(x)\right| & =\frac{1}{(2 \pi)^{d}}\left|\int_{\mathbb{R}^{d}} f(x-t)\left(1_{(-\pi, \pi)^{d}} K_{n}^{\alpha}\right)(t) d t\right| \\
& =\frac{1}{(2 \pi)^{d}} \sum_{k_{1}=-\infty}^{\infty} \cdots \sum_{k_{d}=-\infty}^{\infty} \int_{P_{k_{1}}\left(n_{1}\right)} \cdots \int_{P_{k_{d}}\left(n_{d}\right)}|f(x-t)|\left|\left(1_{(-\pi, \pi)^{d}} K_{n}^{\alpha}\right)(t)\right| d t,
\end{aligned}
$$

where

$$
P_{k_{j}}\left(n_{j}\right):=\left\{x \in \mathbb{R}: 2^{k_{j}-1} \pi / n_{j} \leq|x|<2^{k_{j}} \pi / n_{j}\right\} \quad(j=1, \ldots, d) .
$$


Then,

$$
\begin{aligned}
\left|\sigma_{n}^{\alpha} f(x)\right| & \leq \frac{1}{(2 \pi)^{d}} \sum_{k_{1}=-\infty}^{\infty} \cdots \sum_{k_{d}=-\infty}^{\infty} \int_{P_{k_{1}}\left(n_{1}\right)} \cdots \int_{P_{k_{d}}\left(n_{d}\right)}|f(x-t)| d t \\
& \times \sup _{t \in P_{k_{1}}\left(n_{1}\right) \times \cdots \times P_{k_{d}}\left(n_{d}\right)}\left|\left(1_{(-\pi, \pi)^{d}} K_{n}^{\alpha}\right)(t)\right| \\
& =\frac{1}{(2 \pi)^{d}} \sum_{k_{1}=-\infty}^{\infty} \cdots \sum_{k_{d}=-\infty}^{\infty} \int_{P_{k_{1}}\left(n_{1}\right)} \cdots \int_{P_{k_{d}}\left(n_{d}\right)}|f(x-t)| d t \\
& \times \sup _{t \in P_{k_{1}} \times \cdots \times P_{k_{d}}}\left|\left(1_{(-\pi, \pi)^{d}} K_{n}^{\alpha}\right)\left(\frac{t_{1}}{n_{1}}, \ldots, \frac{t_{d}}{n_{d}}\right)\right| .
\end{aligned}
$$

Consequently, by (4.4),

$$
\begin{aligned}
\left|\sigma_{n}^{\alpha} f(x)\right| & \leq \frac{1}{(2 \pi)^{d}} \sum_{k_{1}=-\infty}^{\infty} \cdots \sum_{k_{d}=-\infty}^{\infty} 2^{k_{1}+\ldots+k_{d}} M_{s} f(x) \sup _{t \in P_{k}}\left|h^{\alpha}(t)\right| \\
& =C\left\|h^{\alpha}\right\|_{E_{\infty}\left(\mathbb{R}^{d}\right)} M_{s} f(x) .
\end{aligned}
$$

Inequality (4.5) finishes the proof.

Inequalities (2.1) and (2.2) imply:

Corollary 4.1. Suppose that $0<\alpha_{j} \leq 1$ for all $j=1, \ldots$, d. If $f \in L_{1}(\log L)^{d-1}\left(\mathbb{T}^{d}\right)$, then

$$
\sup _{\rho>0} \rho \lambda\left(\sigma_{*}^{\alpha} f>\rho\right) \leq C+C\|f\|_{L_{1}(\log L)^{d-1}} .
$$

If $1<p \leq \infty$ and $f \in L_{p}\left(\mathbb{T}^{d}\right)$, then

$$
\left\|\sigma_{*}^{\alpha} f\right\|_{p} \leq C_{p}\|f\|_{p}
$$

The usual density argument due to Marcinkiewicz and Zygmund [12] implies:

Corollary 4.2. Suppose that $0<\alpha_{j} \leq 1$ for all $j=1, \ldots$, d. If $f \in L_{1}(\log L)^{d-1}\left(\mathbb{T}^{d}\right)$, then

$$
\lim _{n \rightarrow \infty} \sigma_{n}^{\alpha} f=f \quad \text { a.e. . }
$$

In this paper, $n \rightarrow \infty$ means that $n_{j} \rightarrow \infty$ for all $j=1, \ldots, d$. Now, we prove that the convergence in Corollary 4.2 holds at each strong Lebesgue point, whenever the corresponding strong Hardy-Littlewood maximal function is finite.

Theorem 4.3. Suppose that $0<\alpha_{j} \leq 1$ for all $j=1, \ldots, d$. If $M_{s} f(x)$ is finite and $x$ is a strong Lebesgue point of $f \in L_{1}(\log L)^{d-1}\left(\mathbb{T}^{d}\right)$, then

$$
\lim _{n \rightarrow \infty} \sigma_{n}^{\alpha} f(x)=f(x) .
$$

Proof. Let

$$
G(u):=\int_{-u_{1}}^{u_{1}} \cdots \int_{-u_{d}}^{u_{d}}|f(x-t)-f(x)| d t \quad\left(u \in \mathbb{R}_{+}^{d}\right) .
$$

Since $x$ is a strong Lebesgue point of $f$, for all $\epsilon>0$, we can find an integer $m \leq 0$ such that

$$
\frac{G(u)}{\prod_{j=1}^{d}\left(2 u_{j}\right)} \leq \epsilon \quad \text { if } \quad 0<u_{j} \leq 2^{m} \pi, j=1, \ldots, d .
$$

Since

$$
\frac{1}{(2 \pi)^{d}} \int_{\mathbb{T}^{d}} K_{n}^{\alpha}(t) d t=1
$$


we have

$$
\left|\sigma_{n}^{\alpha} f(x)-f(x)\right| \leq \frac{1}{(2 \pi)^{d}} \int_{\mathbb{R}^{d}}|f(x-t)-f(x)|\left|\left(1_{(-\pi, \pi)^{d}} K_{n}^{\alpha}\right)(t)\right| d t:=A_{1}(x)+A_{2}(x),
$$

where

$$
\begin{aligned}
A_{1}(x) & :=\frac{1}{(2 \pi)^{d}} \sum_{k_{1}=-\infty}^{m+\left\lfloor\log _{2} n_{1}\right\rfloor} \cdots \sum_{k_{d}=-\infty}^{m+\left\lfloor\log _{2} n_{d}\right\rfloor} \\
& \times \int_{P_{k_{1}}\left(n_{1}\right)} \cdots \int_{P_{k_{d}}\left(n_{d}\right)}|f(x-t)-f(x)|\left|\left(1_{(-\pi, \pi)^{d}} K_{n}^{\alpha}\right)(t)\right| d t
\end{aligned}
$$

and

$$
\begin{aligned}
A_{2}(x) & :=\frac{1}{(2 \pi)^{d}} \sum_{\pi_{1}, \ldots, \pi_{d}} \sum_{k_{\pi_{1}}=m+\left\lfloor\log _{2} n_{\pi_{1}}\right\rfloor+1}^{\infty} \ldots \sum_{k_{\pi_{j}}=m+\left\lfloor\log _{2} n_{\pi_{j}}\right\rfloor+1}^{\infty} \sum_{k_{\pi_{j+1}}=-\infty}^{\infty} \ldots \sum_{k_{\pi_{d}}=-\infty}^{\infty} \\
& \times \int_{P_{k_{1}}\left(n_{1}\right)} \cdots \int_{P_{k_{d}}\left(n_{d}\right)}|f(x-t)-f(x)|\left|\left(1_{(-\pi, \pi)^{d}} K_{n}^{\alpha}\right)(t)\right| d t .
\end{aligned}
$$

Here $\left\{\pi_{1}, \ldots, \pi_{d}\right\}$ is a permutation of $\{1, \ldots, d\}$ and $1 \leq j \leq d$. As in (4.6),

$$
\begin{aligned}
A_{1}(x) & \leq C \sum_{k_{1}=-\infty}^{m+\left\lfloor\log _{2} n_{1}\right\rfloor} \cdots \sum_{k_{d}=-\infty}^{m+\left\lfloor\log _{2} n_{d}\right\rfloor} \int_{P_{k_{1}}\left(n_{1}\right)} \cdots \int_{P_{k_{d}}\left(n_{d}\right)}|f(x-t)-f(x)| d t \\
& \times \sup _{t \in P_{k_{1}} \times \cdots \times P_{k_{d}}}\left|\left(1_{(-\pi, \pi)^{d}} K_{n}^{\alpha}\right)\left(\frac{t_{1}}{n_{1}}, \ldots, \frac{t_{d}}{n_{d}}\right)\right| \\
& \leq C \sum_{k_{1}=-\infty}^{m+\left\lfloor\log _{2} n_{1}\right\rfloor} \cdots \sum_{k_{d}=-\infty}^{m+\left\lfloor\log _{2} n_{d}\right\rfloor} G\left(\frac{2^{k_{1}} \pi}{n_{1}}, \ldots, \frac{2^{k_{d}} \pi}{n_{d}}\right)\left(\prod_{j=1}^{d} n_{j}\right) \sup _{t \in P_{k}}\left|h^{\alpha}(t)\right| .
\end{aligned}
$$

Inequalities (4.7), (4.5) and $2^{k_{j}} / n_{j} \leq 2^{m}$ imply

$$
A_{1}(x) \leq C \epsilon \sum_{k_{1}=-\infty}^{m+\left\lfloor\log _{2} n_{1}\right\rfloor} \cdots \sum_{k_{d}=-\infty}^{m+\left\lfloor\log _{2} n_{d}\right\rfloor} 2^{k_{1}+\ldots+k_{d}} \sup _{t \in P_{k}}\left|h^{\alpha}(t)\right| \leq C \epsilon\left\|h^{\alpha}\right\|_{E_{\infty}\left(\mathbb{R}^{d}\right)} \leq C_{\alpha} \epsilon .
$$

Similarly,

$$
\begin{aligned}
A_{2}(x) & \leq C \sum_{\pi_{1}, \ldots, \pi_{d}} \sum_{k_{\pi_{1}}=m+\left\lfloor\log _{2} n_{\pi_{1}}\right\rfloor+1}^{\infty} \ldots \sum_{k_{\pi_{j}}=m+\left\lfloor\log _{2}\right.}^{\infty} \sum_{\left.n_{\pi_{j}}\right\rfloor+1}^{\infty} \ldots \sum_{k_{\pi_{j+1}}=-\infty}^{\infty} \ldots \sum_{k_{\pi_{d}}=-\infty}^{\infty}|f(x-t)-f(x)| d t\left(\prod_{j=1}^{d} n_{j}\right) \sup _{t \in P_{k}}\left|h^{\alpha}(t)\right| \\
& \times \int_{P_{k_{1}}\left(n_{1}\right)} \cdots \int_{P_{k_{d}}\left(n_{d}\right)} \sum_{\pi_{\pi_{1}, \ldots, \pi_{d}}}^{\infty} \sum_{k_{\pi_{1}}=m+\left\lfloor\log _{2} n_{\pi_{1}}\right\rfloor+1}^{\infty} \ldots \sum_{k_{\pi_{j}}=m+\left\lfloor\log _{2} n_{\pi_{j}}\right\rfloor+1}^{\infty} \ldots \sum_{k_{\pi_{j+1}}=-\infty}^{\infty} \\
& \leq C_{p} \sum_{k_{\pi_{d}}=-\infty} 2^{k_{1}+\ldots+k_{d}} \sup _{t \in P_{k}}\left|h^{\alpha}(t)\right|\left(M_{s} f(x)+|f(x)|\right) .
\end{aligned}
$$

Since $M_{s} f(x)$ and $f(x)$ are finite, the fact $\left\lfloor\log _{2} n_{\pi_{j}}\right\rfloor \rightarrow \infty$ as $T \rightarrow \infty$ imply that $A_{2}(x) \rightarrow 0$ as $n \rightarrow \infty$. 
In the one-dimensional case, if $x$ is a strong Lebesgue point, then $M_{s} f(x)$ is finite and $L_{1}(\log L)^{d-1}\left(\mathbb{T}^{d}\right)=L_{1}\left(\mathbb{T}^{d}\right)$, hence we get back the results due to Lebesgue [11] and Riesz [15] mentioned in the introduction. Recall that $L_{1}(\log L)^{d-1}\left(\mathbb{T}^{d}\right) \supset L_{p}\left(\mathbb{T}^{d}\right)$ for $1<p \leq \infty$ and $d>1$. Since by Theorem 2.1 and (2.1) almost every point is a strong Lebesgue point and the strong maximal operator $M_{s} f$ is almost everywhere finite for $f \in L_{1}(\log L)^{d-1}\left(\mathbb{T}^{d}\right)$, Theorem 4.3 implies Corollary 4.2. If $f$ is continuous at a point $x$, then $x$ is also a strong Lebesgue point. So we obtain:

Corollary 4.3. Suppose that $0<\alpha_{j} \leq 1$ for all $j=1, \ldots, d$. If $M_{s} f(x)$ is finite and $f \in L_{1}(\log L)^{d-1}\left(\mathbb{T}^{d}\right)$ is continuous at a point $x$, then

$$
\lim _{n \rightarrow \infty} \sigma_{n}^{\alpha} f(x)=f(x) .
$$

\section{REFERENCES}

[1] S. Y. A. Chang, R. Fefferman: Some recent developments in Fourier analysis and $H^{p}$-theory on product domains, Bull. Amer. Math. Soc., 12 (1985), 1-43.

[2] H. G. Feichtinger, F. Weisz: Wiener amalgams and pointwise summability of Fourier transforms and Fourier series, Math. Proc. Cambridge Philos. Soc., 140 (2006), 509-536.

[3] L. Fejér: Untersuchungen über Fouriersche Reihen, Math. Ann., 58 (1904), 51-69.

[4] G. Gát: Pointwise convergence of cone-like restricted two-dimensional $(C, 1)$ means of trigonometric Fourier series, J. Approx. Theory., 149 (2007), 74-102.

[5] G. Gát: Almost everywhere convergence of sequences of Cesàro and Riesz means of integrable functions with respect to the multidimensional Walsh system, Acta Math. Sin., 30 (2) (2014), 311-322.

[6] G. Gát, U. Goginava and K. Nagy: On the Marcinkiewicz-Fejér means of double Fourier series with respect to WalshKaczmarz system, Studia Sci. Math. Hungar., 46 (2009), 399-421.

[7] U. Goginava: Marcinkiewicz-Fejér means of d-dimensional Walsh-Fourier series, J. Math. Anal. Appl., 307 (2005), $206-$ 218.

[8] U. Goginava: Almost everywhere convergence of $(C, \alpha)$-means of cubical partial sums of d-dimensional Walsh-Fourier series, J. Approx. Theory, 141 (2006), 8-28.

[9] U. Goginava: The maximal operator of the Marcinkiewicz-Fejér means of d-dimensional Walsh-Fourier series, East J. Approx., 12 (2006), 295-302.

[10] B. Jessen, J. Marcinkiewicz and A. Zygmund: Note on the differentiability of multiple integrals, Fundam. Math., 25 (1935), 217-234.

[11] H. Lebesgue: Recherches sur la convergence des séries de Fourier, Math. Ann., 61 (1905), 251-280.

[12] J. Marcinkiewicz, A. Zygmund: On the summability of double Fourier series, Fund. Math., 32 (1939), 122-132.

[13] K. Nagy, G. Tephnadze: The Walsh-Kaczmarz-Marcinkiewicz means and Hardy spaces, Acta Math. Hungar., 149 (2016), 346-374.

[14] L. E. Persson, G. Tephnadze and P. Wall: Maximal operators of Vilenkin-Nörlund means, J. Fourier Anal. Appl., 21 (1) (2015), 76-94.

[15] M. Riesz: Sur la sommation des séries de Fourier, Acta Sci. Math. (Szeged), 1 (1923), 104-113.

[16] S. Saks: Remark on the differentiability of the Lebesgue indefinite integral, Fundam. Math., 22 (1934) 257-261.

[17] P. Simon: Cesàro summability with respect to two-parameter Walsh systems, Monatsh. Math., 131 (2000), 321-334.

[18] P. Simon: $(C, \alpha)$ summability of Walsh-Kaczmarz-Fourier series, J. Approx. Theory, 127 (2004), 39-60.

[19] F. Weisz: Summability of Multi-dimensional Fourier Series and Hardy Spaces, Mathematics and Its Applications, Kluwer Academic Publishers, Dordrecht, Boston, London, (2002).

[20] F. Weisz: Summability of multi-dimensional trigonometric Fourier series, Surv. Approx. Theory, 7 (2012), 1-179, .

[21] A. Zygmund: Trigonometric Series. Cambridge Press, London, 3rd edition, (2002).

FERENC WEISZ

EÖTVÖS L. UNIVERSITY

DEPARTMENT OF NUMERICAL ANALYSIS

H-1117 BudAPEST, PÁZMÁNY P. SÉTÁNY 1/C., Hungary

ORCID: 0000-0002-7766-2745

E-mail address: weisz@inf.elte.hu 